\title{
On the topology of the exceptional varieties in birational transformations.
}

\author{
Memoria di Heinrich Guggenheimer (a Ramat Gan, Israel) (*)
}

Suuto - $\dot{E}$ dato dall'Introduzione.

\section{INTRODUOTION}

A birational transformation between two classical algebraic varieties $V$ and $V^{\prime}$ of same dimensions is given by an algebraic subvariety $W \subset V \times V^{\prime}$ such that the projections

$$
\begin{aligned}
& p r_{1}: W \longrightarrow V \\
& p r_{2}: W \longrightarrow V^{\prime}
\end{aligned}
$$

are analytical mappings of degree of mapping one (all algebraic varieties are orientable pseudomanifolds, hence the Brouwer degree is defined by simplicial approximations). Since a complex-analytical mapping defines no coverings with negative indices, the projections must be one-to-one except probably on analytic subcomplexes of (simplicial) dimension at most $n-2$, if $n$ is the dimension of the surrounding complex. We define the exceptional varieties of the birational transformation to be the minimal algebraic subvarieties $M \subset V, N \subset W, M^{\prime} \subset V^{\prime}$ such that the analytical mappings

$$
\begin{aligned}
& p r_{1}: W-N \rightarrow V-M \\
& p r_{2}: W-N \rightarrow V^{\prime}-M^{\prime}
\end{aligned}
$$

are one-to-one onto. In the classical language, $M$ and $M^{\prime}$ are both the union of the fundamental subvariety in one variety and of the image under the transformation of the fundamental subvariety of the other variety. We want to investigate the relations between the homology properties of the six algebraic varieties $V, V^{\prime}, W, M, M M^{\prime}, N$, in the classical case (complex field) only. In the case of Cremona transformations $\left(V \neq V^{\prime}=\right.$ the complex projective space of a given dimension), the problem has recently been solved [1], also for the relations between the torsion coefficients.

A first approximation to our problem is given by the topological theory of modifications. Two spaces $X, Y$ (triangulable, of finite dimension) are said to be modifications of one another, if there exist subspaces $M \subset X, N \subset Y$

(*) L'autore è ora a Pullman (Wash.; U.S.A). 
such that $X-M$ and $Y-N$ are homeomorphic. If this homeomorphism is the restriction of a continuous mapping

$$
\varphi: X \rightarrow Y
$$

we have a mapping-modification (a notion introduced by AEPPLI). A birational transformation is thus generated by two mapping-modifications working in two different directions.

A great number of results are known on modifications [2,3]. Unfortunately, the topological assumptions in the theorems are too restrictive as to make them immediately applicable to algebraic rarieties, since even in the case of non-singular varieties $V, V^{\prime}$ (i. e. topological manifolds), the varieties $W, M, M, N$ will present all kinds of singularities. It is the purpose of this Note to treat some cases of effective situations in birational geometry, though the general case will have to be dealt with by other methods [4].

In the first part, we deal with birational transformations of non-singular manifolds in the general case, i. e. $W, M, M^{\prime}, N$ may possess any kind of algebraic singularity. The results are obtained for rational coefficients, so they represent relations between the BETTI-numbers of the spaces involved. The case of integer coefficients is not treated, but the example of the CREMONA transformations shows that no simple results can be expected for relations between torsion coefficients.

One of the central problems of algebraic geometry is the resolution of singularities. Most of the attempts, successful or abortive, in this field in fact do not deal with general birational transformations between singular and non-singular varieties, but they attempt to «explode» the singularities, as in the case of the dilations used by B. SEgRE. If $Y$ is a variety, and $X$ the variety derived from $Y$ by dilation of a subvariety of $Y$, then by collapsing the dilation we get a mapping modification

$$
\varphi: X \rightarrow Y \text {. }
$$

In the second part of this paper we treat birational transformations under the additional hypothesis, that every variety is the image under an analytical map of a non-singular algebraic variety. Since so many futile attempts have been made to prove this conjecture, the principles of mathematical methodology demand that one should now try to construct a counterexample in order to disprove it. A counterexample of this kind conld be given not only by a direct proof that a given variety is not the projection of a non-singular model (such a proof would probably be most difficult), but it would be sufficient to exhibit a birational transformation whose exceptional varieties do not ful. fill the homological relations derived by the use of the hypothesis.

In the second part, there appear some diagrams that are rather complicated to write down. 
In order not to be troubled by too much short exact sequences, we will use the following notation of group homomorphisms:

$$
\begin{array}{ll}
\text { homomorphism (into or onto): } & \longrightarrow \\
\text { homomorphism onto } & :-1 \\
\text { isomorphism (into or onto) } & := \\
\text { isomorphism onto } & :=
\end{array}
$$

Throughout the paper, the following notation will be used: «variety» is an algebraic variety in some complex projective space, "manifold» is an algebraic variety that is a topological manifold, i.e. without singularities. No effort will be made to extend our results to general analytical spaces.

\section{Maps of pseudomanifolds onto manifolds.}

As a preliminary problem, let us consider a mapping-modification of an orientable closed pseudomanifold $W$ and an orientable closed manifold $V$ :

$$
\varphi: W \rightarrow V
$$

where the dimensions of $W$ and $V$ are supposed to be equal, say $n$, and the dimension of the singular subspaces $N \subset W, M \subset V$ are both $<n$. By definition, $\varphi$ is an orientation-preserving mapping of degree one.

All cohomology groups are on the rationals as coefficients, so we suppress the mention of the group of coefficients in the symbols of cohomology groups. Since $\varphi$ is continuous, $M$ and $N$ are closed, and it is well known that the simplicial groups of a compact space modulo a closed subspace are naturally isomorphic tho the singular groups with compact carriers on the open difference space, so they depend only on this difference space. Since the restriction

$$
\varphi: W-N \rightarrow V-M
$$

is a homeomorphism, the exact sequences of $(W, N)$ and $(V, M)$ are connected by the inverse homomorphism to $\varphi$ :

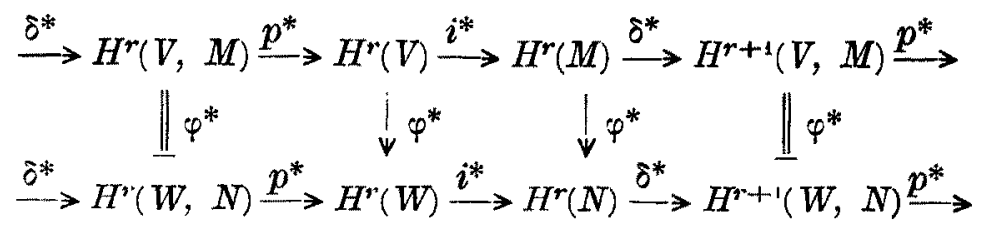

This situation has been treated by AEPPLI [2] under the assumption that both $W$ and $V$ are closed orientable manifolds. We show that nothing is changed 
in his results if $W$ is a closed orientable pseudomanifold. (One may see by simple twodimensional examples that the situation is thoroughly changed if $V$ is a pseudomanifold with singularities).

First we say that in fact we have an isomorphism

$$
\varphi^{*} ; H^{\prime}(V) \Longrightarrow H^{\prime}(W)
$$

or that, given any non-zero element $\zeta^{*} \in H^{*}(V)$, its image $f^{*} \zeta^{r} \neq 0$. Let $\mu$ be the generator of $H^{n}(V)$, w that of $H^{\prime \prime}(W)$, both uniquely defined by the orien. tation, and $(\varphi$ is or degree one!)

$$
\varphi^{*} \mu=\omega .
$$

On $V$, harmonic rational chains are both cycles and cocycles, hence to $\zeta^{r}$ corresponds by PoINCARÉ's duality a unique $\zeta^{n-r}$ with $\zeta^{r} \cup \zeta^{n-r}=\mu$. But

so

$$
\varphi^{*}\left(\zeta^{*} \cup \zeta^{n-r}\right)=\varphi^{*} \zeta^{*} \cup \varphi^{*} \zeta^{n-r}=\omega \neq 0
$$

$$
\varphi^{*} \zeta \mathbf{r} \neq 0 \text {. }
$$

The quadratic diagrams connecting the exact sequences derive from space maps, hence they are commutative and one sees by a simple argument on the dimensions of the vector-spaces $H^{r}(M), H^{r}(N)$, that also

$$
\varphi^{*}: H^{r}(M) \Longrightarrow H^{r}(N) \text {. }
$$

From the exact and commutative diagram

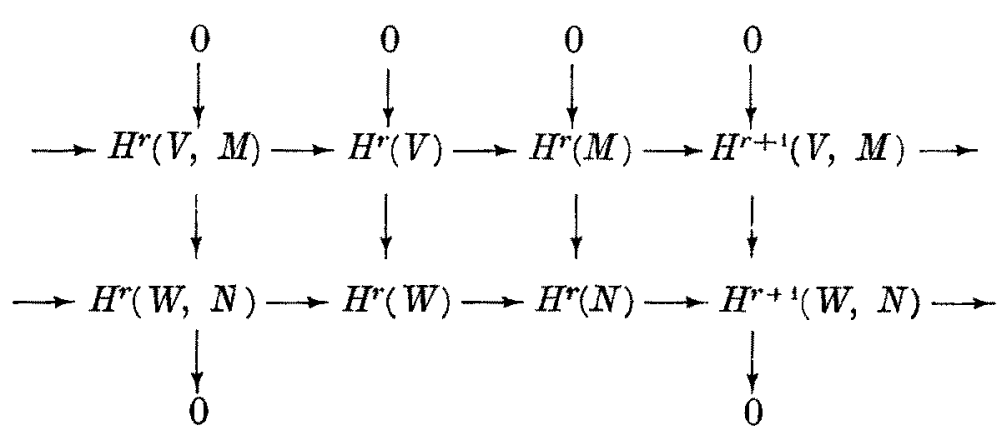

one has, as in [2]:

$$
H^{r}(V) / H^{r}(M) \cong H^{r}(W) /{ }^{r} H(N),
$$

or for the BerTi-numbers $b_{r}(X)=\operatorname{dim} H^{r}(X)$,

$$
b_{r}(V)-b_{r}(M)=b,(W)-b_{r}(N)
$$

In the case of a birational transformation, we have likewise

$$
b_{r}\left(V^{\prime}\right)-b_{r}\left(M^{\prime}\right)=b_{r}(W)-b_{r}(N),
$$


H. GUGGENHenMER: On the topology of the exceptional varieties, etc.

hence the relation

$$
b_{r}(V)-b_{r}(M)=b_{r}\left(V^{\prime}\right)-b_{r}\left(M^{\prime}\right)
$$

holds for every birational transformation between non-singular varieties, without any restriction on the transformation $W$.

For algebraic curves, ZeUTHeN's formula shows that (2) is true even if $V$ and $V^{\prime}$ have singalarities, since in that case antomatically $b_{r}(V)=b,\left(V^{\prime}\right)$, $b,(M)=b, r(M)=0$ for $r>0$, and $b_{0}(\boldsymbol{M}), b_{0}\left(\boldsymbol{M}^{\prime}\right)$ are the number of brach-points of $W$ over $V, V^{\prime}$ respectively.

\section{Varieties with singularities.}

In this paragraph, we will deal with birational transformations of singular varieties under the hypothesis that every algebraic variety may be transformed into a manifold by a finite number of dilations [5]. A dilation $D(V)$ of a variety $V$ in a subvariety $M$ may be described by a map

$$
d: D(V) \rightarrow V
$$

continuous everywhere, such that the restriction

$$
d: D(V)-D(M)-V-M
$$

is a homeomorphism and

$$
d: D(M) \rightarrow M
$$

is a fiber map, the fiber being a complex projective space of (real) dimension $\operatorname{dim} V-\operatorname{dim} M-2$. In order to avoid complications, $M$ should consist of components of one dimension only. The "explosion» of a general reducible variety should be decomposed into dilations of homogeneous-dimensional parts. By lifting

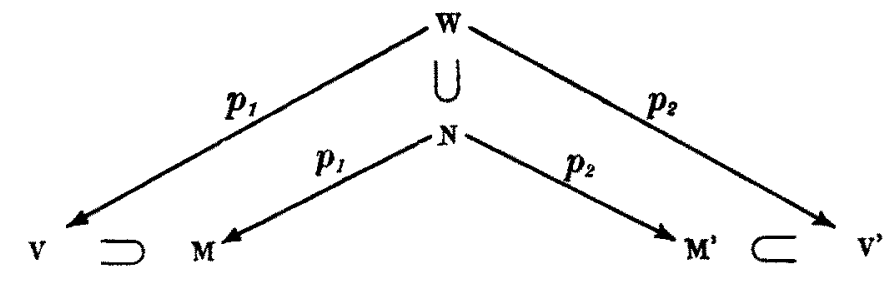

Fig. 1

maps, we get to every birational transformation a birational transformation between the dilated manifolds which covers the original one; where all squares 
are commutative. At a first step, one may apply our hypothesis in orter to get $D(V)$ and $D\left(V^{\prime}\right)$ as manifolds. $D\left(W^{\prime}\right) \subset D(V) \times D\left(V^{\prime}\right)$ is then given, but is not

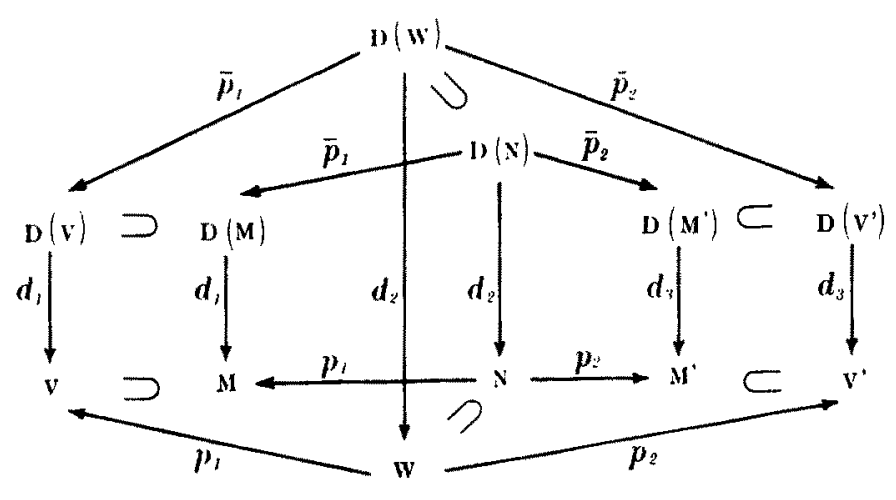

Fig. 2

necessarily a manifold. But here one may apply a new dilation

$$
d_{4}: D D(W) \rightarrow D(W)
$$

such that $D D(W)$ is a manifold, and, putting $\vec{p}_{i} d_{i}=q_{i}, i=1,2$, we have the following commutative diagram, where all boldfaceset spaces are manifolds, but where in general $D D(W) \notin D(V) \times D\left(V^{\prime}\right)$. By our definitions, all maps

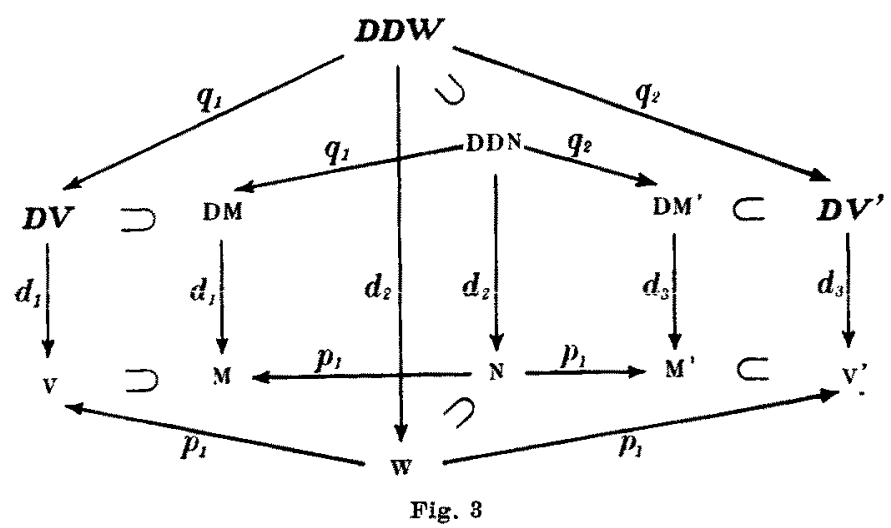

appearing in this diagram are mapping-modifications. Hence we may use the results of the preceding paragraph in order to connect the exact sequences of the couples of spaces not only by homomorphisms, but by isomorphisms. 
We write down the whole diagram for the left part of the diagram of spaces, i. e. involving the mapping-modifications $q_{1}, d_{1}, d_{2}, p_{1}$.

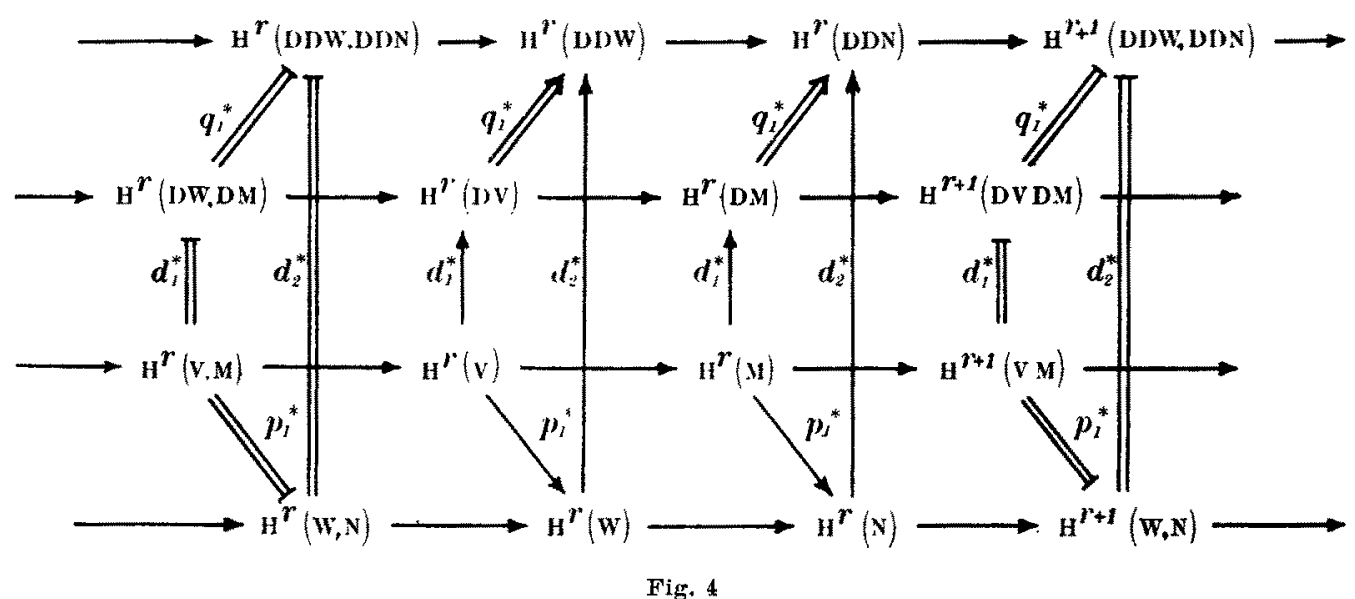

Now we say that in the case of a dilation, all induced homomorphisms are indeed isomorphisms. This follows from the fact [6] that the cohomology groups of $D M$ are those of the topological product of $M$ with a complex projective space, where this complex projective space is represented by a nonvanishing cocycle in $V$ (in fact, the dual of an algebraic cycle). Therefore [6] $H^{*}(D V)$ contains an isomorphic image of $H^{*}(V)$. But in this case it follows from the commutativity of the space-maps that all homomorphisms $p_{1}{ }^{*}$ are also isomorphisms. As in the preceding case, we have

$$
b_{r}(V)-b_{,}(M)=b_{r}(W)-b_{r}(N) \text {. }
$$

Having established this relation, all other pairs of exact sequences yield only a repetition of it, since

$$
b,(D M)=\sum_{j=0}^{[r / 2]} b_{r-2 j}(M)
$$

We again formulate our result:

If the hypothesis is true that all algebraic varieties admit a resolution by dilations, then (2) holds for every birational transformation, even between singular varieties.

From the fact that all homomorphisms in the diagram are isomorphism, we have also:

Under the same hypothesis,

$$
\begin{aligned}
& b_{r}(W) \geq \max \left(b_{r}(V), b_{,}\left(V^{\prime}\right)\right) \\
& \left.b_{r}(N) \geq \max b,(M), b_{r}\left(M^{\prime}\right)\right) .
\end{aligned}
$$


8 H. GUGGENHEIMER: On the topology of the exceptional varieties, etc.

\section{BIBLIOGRAPHY}

[1] B. Segre, Corrispondenze biraeionali e topologia di varietà algebriche, "Ann, di Mat." (14) 43 (1957), 1-23.

[2] H. Guggenheimer, n-dimensional analogue of the Cremona-Clebsch theorem, .Bull. Amer. Math. Soc. , to appear.

[3] A. AgppLI, Modifikationen von reellen und komplexen Mannigfaltigkeiten, "Comm. Math. Helv. . 31, 1956, 219-301.

[4] H. GUGGEnhemmer, Opératewrs aifférentielles et suites exactes sur une variété diffèrentielle, * Tôhoka J. Math. » (2) 8, 1956, 308 - 323.

Modifications of a manifold in one point, «Math. Japon " IV, 1956. 5-11. Modifications in Real and Complex Curves, "Nath. Japon" IV, 19506, 33-47.

[5] H. Guggenhaimer Saggi di topologia delle varietà complesse, parte IIT, "Rend. Acc. Sei. Bologna * (11) 3, (1955-56), 243 -253.

[6] B. SEgre, Sullo scioglimento delle singolarith delle varietà algebriche, "Ann. di Mat." (4) $33,1952,5-48$.

Dilataeioni e comportamenti associati nel campo analitico, "Rend. Circ. Mat. di Palermo* (2) 1, 1952, 373-379.

[7] H. Gugagnheimer, Omologia delle dilatazioni, *Rend Acc. Naz. Lincei * (8) 17, (19is), $13-15$. 